\title{
Gli uccelli del Parco Regionale della Maremma
}

\author{
a cura di Pietro Giovacchini
}

Vi sono sicuramente buone ragioni per portare a conclusione un progetto come questo.

L'occasione è quella decisiva per ricavarne dalla sua realizzazione quelle informazioni che, in molte circostanze, attendiamo da tempo e che risultano essere essenziali per redigere, ad esempio, elenchi delle specie della flora o fauna delle nostre aree protette. Proteggere significa anche conoscere e l'acquisizione scientifica di base è imprescindibile per garantire la conservazione, valorizzazione e gestione del patrimonio naturale. Gli Uccelli rappresentano il gruppo di vertebrati più numeroso della fauna italiana: ad essi fa riferimento questo lavoro sull'avifauna del Parco Regionale della Maremma e delle zone limitrofe, lavoro che si richiama per ispirazione ad un "modello" di ornitologia ancora oggi indispensabile, di impostazione moltoniana. Un doveroso tributo verso un'area geografica insignita nel 1992, da parte del Consiglio d'Europa, del Diploma Europeo delle aree protette, in cui una grande varietà ambientale ed ecosistemi di assoluta rilevanza nel panorama dell'intera regione biogeografica mediterranea costituiscono parte essenziale dello studio. Questo fascicolo, oltre che raccogliere i risultati di un'esaustiva attività di ricerca bibliografica, archiviazione e selezione delle informazioni radunate per decenni, rappresenta soprattutto la risultante di centinaia e centinaia di giornate passate in ogni luogo del Parco, quanto basta per descrivere la ricchezza specifica sin qui raggiunta nel periodo che va dall'anno 1975, con l' istituzione dell'area protetta, alla conclusione di questa indagine definita con la chiusura del 2018. Sono, infatti, 295 le specie rinvenute complessivamente in questo comprensorio incastonato tra Principina Mare, Palude della Trappola e foce del fiume Ombrone, la pineta, le zone agricole, i pascoli di Alberese e Torre Trappola ed il comprensorio boschivo dei Monti dell'Uccellina sino a Talamone sul mare, lungo circa 25 chilometri di costa.

Non da meno, affinché ciò che si delinea per essere definito un lavoro completo possa anche risultare un unicum nel panorama dell'ornitologia locale, attraverso un interessante contributo di Filippo Ceccolini e Fausto Barbagli si è indagato quel vivace periodo posto a cavallo tra il XIX e inizio XX secolo, portando alla luce una preziosa mole di reperti museali radunati principalmente per la Collezione Centrale degli Animali Vertebrati Italiani, presso il Museo di storia naturale dell'Università degli studi di Firenze, e riferiti anche all'attività di caccia allora praticata principalmente nelle Tenute di Alberese e della Trappola.

Pietro Giovacchini 
L'Ente Parco Regionale della Maremma ha accolto con favore la richiesta di partecipare alla realizzazione di questo fascicolo della prestigiosa Rivista italiana di Ornitologia e, dopo aver letto il lavoro giunto al termine, la decisione di essere tra i finanziatori dell'opera ne esce ulteriormente rafforzata. Non solo per il fatto che il lavoro di gestione e conservazione degli habitat svolto in questi anni dimostra che l'Ente ha intrapreso la giusta direzione - ed i numeri delle specie di uccelli rilevate e della loro varietà ne danno atto in maniera evidente - ma anche perché è estremamente utile, proprio ai fini gestionali, poter usufruire di una rassegna completa ed esaustiva dell'avifauna presente nell'area protetta, sia essa nidificante o di passaggio.

Il fatto poi che molte delle specie presenti siano ricomprese in liste di particolare protezione, conferma l'importanza delle aree protette per la conservazione degli habitat e dunque della biodiversità.

Così come luoghi in cui si possono sperimentare progetti scientifici tesi all'ampliamento della biodiversità, come ha dimostrato il progetto sul Falco pescatore, che è riuscito a far tornare a nidificare non solo all'interno del Parco della Maremma, ma anche in altre aree del territorio maremmano, questa specie che aveva rinunciato, per più di quarant'anni, ad eleggerlo come sito di nidificazione.

Un progetto, che nel corso degli anni ha infatti allargato l'areale di azione anche ad altri Parchi e riserve naturali della Toscana, con l'ambizione di ricreare un'area di nidificazione "stabile" nel bacino del Mediterraneo.

L'auspicio è che si possa continuare nella conservazione degli habitat e fare in modo che la lista già ampia di specie di avifauna presenti ne possa annoverare altre, che trovano nel Parco della Maremma un'area dove sussistono le condizioni ottimali per fare il proprio nido o comunque per trascorrere un periodo del loro ciclo vitale.

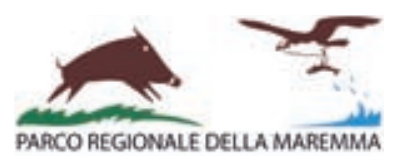


È con grande piacere che andiamo a presentare il fascicolo della Rivista italiana di Ornitologia dedicato agli uccelli del Parco Regionale della Maremma e realizzato dal dr. Pietro Giovacchini. Per noi, sponsor dell'iniziativa, biodiversità e tutela degli elementi naturali, così come delle attività economiche tradizionali collegate all'area protetta, sono il giusto prosieguo di chi ci ha preceduti in questa importante scommessa. Lo è ancor di più perché la Tenuta Torre Trappola si colloca in uno scenario ambientale del tutto unico nel panorama toscano: lo stesso che ci ha resi immediatamente partecipi di quelle emozioni che la Maremma Grossetana è in grado di fornire durante tutto l'anno. Per quanto struggente, è dunque alla memoria della nostra cara Fulvia Ferragamo Visconti che dedichiamo questa pubblicazione perché nella protezione della natura e dei valori produttivi sostenibili che contraddistinguono Torre Trappola ella ha sempre fatto riferimento, evidenziando una crescente e sincera appartenenza a questo territorio. Non da meno, alle tantissime gru, oche, fenicotteri, occhioni, colombelle ed a tutte le altre specie di uccelli che popolano il Parco, ed in particolare la Trappola, curiosità e rispetto ci sono stati di insegnamento grazie anche a Fulvia.

Siamo convinti che gli uccelli saranno dunque lieti di questa sincera attenzione editoriale.

Famiglia Visconti Ferragamo Società Agricola Torre Trappola S.a.s.

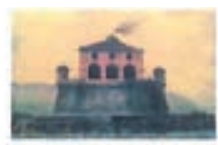

LA. TRAPPOLA 
Il GOM - Gruppo Ornitologico Maremmano - Studi Naturalistici "A. Ademollo" - si è costituito come organizzazione di volontariato nel 2010, dopo aver operato per alcuni anni in provincia di Grosseto come gruppo informale dedito agli studi naturalistici. La finalità sociale dell'organizzazione, ribadita nel nuovo Statuto approvato a seguito della riforma del Terzo Settore, è quella di "promuovere, organizzare e realizzare studi, ricerche e progetti su fauna, flora e ambienti naturali, con particolare riferimento all'avifauna della Provincia di Grosseto, anche in funzione dell'individuazione di strategie e attività di conservazione per le specie minacciate ed i loro habitat".

Il buon numero di soci (attualmente 81), attivi praticamente in tutto il territorio provinciale, permette di radunare un gran numero di osservazioni, derivanti anche da appositi progetti, che vanno a confluire nella Banca Dati dell'associazione.

Ovviamente, proprio per la citata finalità sociale e per consegnare un giusto riconoscimento ad uno strumento fino ad oggi mancante nel panorama delle pubblicazioni scientifiche, il GOM ha voluto contribuire alla realizzazione di questo fascicolo della gloriosa Rivista italiana di Ornitologia dedicato all'avifauna del Parco Regionale della Maremma, sia con un piccolo contributo economico che, soprattutto, mettendo a disposizione dell'autore Pietro Giovacchini, le informazioni presenti nella Banca Dati.

Siamo perciò estremamente soddisfatti di questo importante lavoro portato a termine, auspicandone la più ampia diffusione possibile.

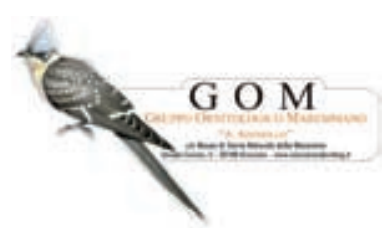

